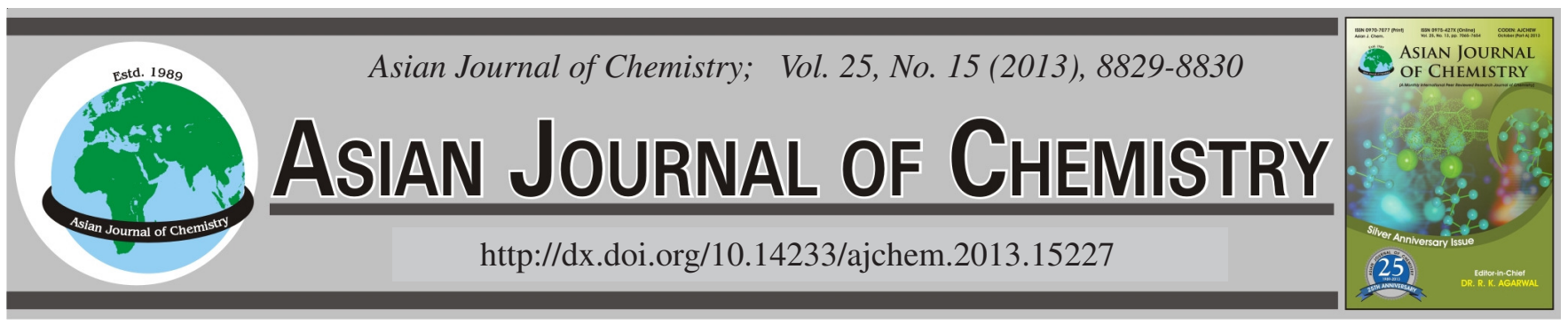

NOTE

\title{
Synthesis and Crystal Structure of Binuclear Ni(II) Complex Derived from 2-Nitrobenzoic Acid and Tetramethylethylenediamine
}

\author{
ADNAN M. QADIR
}

Department of Chemistry, Collage of Science, Salahaddin University, Erbil 44001, Kurdistan Region, Iraq

Corresponding author: E-mail: adnan_qadir2000@yahoo.com

(Received: 28 January 2013;

Accepted: 30 August 2013)

AJC-14059

\begin{abstract}
A nickel(II) complex of the formula $\left[\mathrm{Ni}_{2}(\mathrm{nba})_{4}(\mathrm{tmen})_{2}\left(\mathrm{H}_{2} \mathrm{O}\right)\right] \cdot \mathrm{C}_{2} \mathrm{H}_{5} \mathrm{OH}(\mathbf{1})$, where nba $=2$-nitrobenzoate, tmen = tetramethylethylenediamine, has been synthesized and determined by single crystal X-ray diffraction method. The crystal belongs to monoclinic system, space group P121/c1 with cell dimensions of $a=11.3931$ (4), $b=33.0702$ (4), $c=13.5623$ (4) $\AA, \alpha=90, \beta=111.310$ (4) and $\gamma=90^{\circ}$. The two nickel atoms are connected by one bridging water molecule and two bidentate bridging carboxylate ligands. The octahedral environment around each nickel atom is completed by two nitrogen atoms from tetramethylethylenediamine and one oxygen atom from monodentate carboxylate.
\end{abstract}

Key Words: 2-Nitrobenzoic acid, Nickel, Crystal, Tetramethylethylenediamine.

The versatility of carboxylate ligand is reflected by variety of binding modes. It can coordinate as monodentate, bidentate, monodentate bridging, bidentate bridging, etc. The metal carboxylates play an important role in synthetic chemistry, biological and catalytic activities and physiological effect $^{1,2}$. Among these carboxylate complexes, nickel representatives have attracted great attention since nickel has been recognized as a considerable important biological agent forming the active site of variety of metalloproteins ${ }^{3,4}$. In addition, their adducts with nitrogen have potential applications in area of organic conductor and magnetic materials ${ }^{5}$. In this paper, we reported the synthesis and crystal structure of $\left[\mathrm{Ni}_{2}(\mathrm{nba})_{4}(\text { tmen })_{2}\left(\mathrm{H}_{2} \mathrm{O}\right)\right] \cdot \mathrm{C}_{2} \mathrm{H}_{5} \mathrm{OH}$, where nba = 2-nitrobenzoate, tmen $=$ tetramethylethylenediamine.

Synthesis: To ethanolic solution $(30 \mathrm{~mL})$ of $\mathrm{NiCl}_{2} \cdot 6 \mathrm{H}_{2} \mathrm{O}$ $(0.48 \mathrm{~g}, 2 \mathrm{mmol})$, was added ethanolic solution $(30 \mathrm{~mL})$ of 2-nitrobenzoate $(0.67 \mathrm{~g}, 4 \mathrm{mmol})$ followed by tetramethylethylenediamine $(0.23 \mathrm{~g}, 2 \mathrm{mmol})$. Catalytic amount of $\mathrm{Et}_{3} \mathrm{~N}$ was also added. The mixture was refluxed for $1 \mathrm{~h}$. Single crystal suitable for X-ray crystallographic analysis was obtained by slow evaporation at room temperature after 3 days.

Structure determination: A single crystal of the present $\mathrm{Ni}$ (II) complex with dimensions of $0.2933 \mathrm{~mm} \times 0.2052 \mathrm{~mm}$ $\times 0.0623 \mathrm{~mm}$ was mounted with paratone on a glass needle and the data were collected on an Oxford Diffraction SuperNovaarea-detector diffractometer with mirror optics monochromated $\mathrm{MoK}_{\alpha}$ radiation $(\lambda=0.71073 \AA)$ using $\omega$ scan mode at $173 \mathrm{~K}$. The structure was solved by direct methods using SIR $97^{6}$. All H-atoms were placed in geometrically calculated positions. Refinement of the structure was carried out on $\mathrm{F}^{2}$ using full-matrix least-squares procedures. All calculations were performed using the SHELXL-97 ${ }^{6,7}$ program. Crystal data and refinement for complex $\mathbf{1}$ are listed in Table-1.

A molecular structure with atoms labeling scheme of complex 1 are shown in Fig. 1. Selected bond lengths and bond angles are listed in Table- 2 .

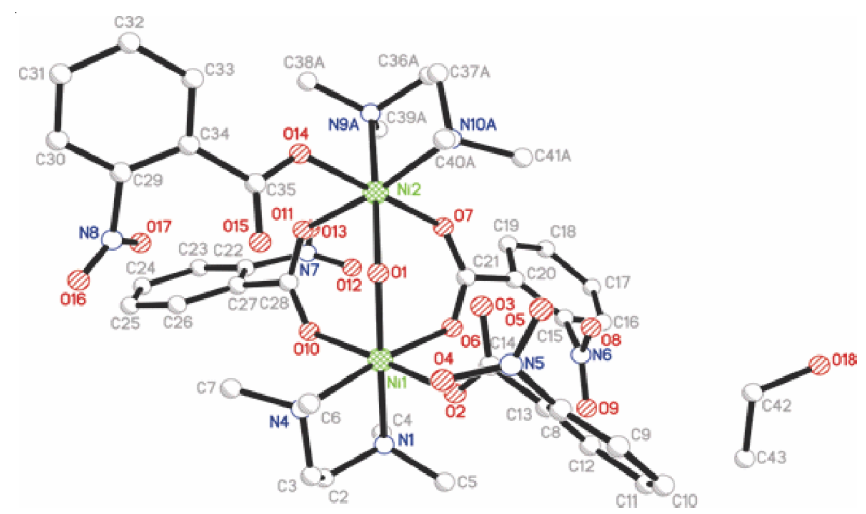

Fig. 1. Molecular structure of complex $\mathbf{1}$

In the structure of $\left[\mathrm{Ni}_{2}(\mathrm{nba})_{4}(\mathrm{tmen})_{2}\left(\mathrm{H}_{2} \mathrm{O}\right)\right] \cdot \mathrm{C}_{2} \mathrm{H}_{5} \mathrm{OH}$ each nickel atom is six coordinated by two nitrogen atoms from tmen, three oxygen atoms from three nba ligands and one oxygen atom from water molecule showing distorted octahedral geometry. Two coordination modes of carboxylate ligands are 


\begin{tabular}{|c|c|c|c|}
\hline & & \multicolumn{2}{|c|}{ CRYSTAL DATA AND STRUCTL } \\
\hline \multicolumn{2}{|c|}{ Empirical formula } & \multicolumn{2}{|c|}{$\mathrm{C}_{42} \mathrm{H}_{56} \mathrm{~N}_{8} \mathrm{O}_{18} \mathrm{Ni}_{2}$} \\
\hline \multicolumn{2}{|c|}{ Space group } & \multicolumn{2}{|c|}{$\mathrm{P} 121 / \mathrm{c} 1$} \\
\hline \multicolumn{2}{|c|}{$\mathrm{a}(\AA)$} & \multicolumn{2}{|c|}{$11.3931(4)$} \\
\hline \multicolumn{2}{|c|}{$\mathrm{b}(\AA)$} & \multicolumn{2}{|c|}{$33.0702(4)$} \\
\hline \multicolumn{2}{|c|}{$\mathrm{c}(\AA)$} & & $13.5623(4)$ \\
\hline \multicolumn{2}{|c|}{ Volume $(\AA)^{3}$} & \multicolumn{2}{|c|}{$4760.5(2)$} \\
\hline \multicolumn{2}{|c|}{$\mathrm{D}_{\mathrm{c}}\left(\mathrm{Mg} / \mathrm{m}^{3}\right)$} & \multicolumn{2}{|c|}{1.505} \\
\hline \multicolumn{2}{|c|}{$\mathrm{F}_{(000)}$} & \multicolumn{2}{|c|}{22.56} \\
\hline \multicolumn{2}{|c|}{$\mathrm{h}, \mathrm{k}, \mathrm{l}$ ranges } & \multicolumn{2}{|c|}{$-1514,-4342,-1718$} \\
\hline \multirow{2}{*}{\multicolumn{2}{|c|}{$\begin{array}{l}\text { Data/restraints/parameters on } \mathrm{F}^{2} \\
\mathrm{R} \text { indices }[\mathrm{I}>2 \sigma(\mathrm{I})] \\
\text { Largest diff. peak and hole }\left(\AA^{-3}\right)\end{array}$}} & \multicolumn{2}{|c|}{$10855 / 133 / 699$} \\
\hline & & \multicolumn{2}{|c|}{$\begin{array}{c}\mathrm{R}_{1}=0.0719, \mathrm{wR}_{2}=0.2009 \\
0.989 \text { and }-0.833\end{array}$} \\
\hline \multicolumn{4}{|c|}{$\begin{array}{c}\text { TABLE- } 2 \\
\text { SELECTED BOND DITANCES ( }(\AA) \\
\text { AND ANGLES }\left({ }^{\circ}\right) \text { FOR COMPLEX } 1\end{array}$} \\
\hline $\mathrm{Ni}(1)-\mathrm{O}(10)$ & $2.042(3)$ & $\mathrm{Ni}(1)-\mathrm{O}(2)$ & $2.059(4)$ \\
\hline $\mathrm{Ni}(1)-\mathrm{O}(6)$ & $2.034(3)$ & $\mathrm{Ni}(1)-\mathrm{N}(1)$ & $2.160(4)$ \\
\hline $\mathrm{Ni}(2)-\mathrm{N}(9 \mathrm{~A})$ & $2.155(14)$ & $\mathrm{Ni}(2)-\mathrm{O}(1)$ & $2.119(3)$ \\
\hline $\mathrm{Ni}(2)-\mathrm{N}(10 \mathrm{~A})$ & $2.165(14)$ & $\mathrm{Ni}(2)-\mathrm{O}(11)$ & $2.040(3)$ \\
\hline $\mathrm{O}(10)-\mathrm{Ni}(1)-\mathrm{N}(4)$ & $90.03(15)$ & $\mathrm{O}(6)-\mathrm{Ni}(1)-\mathrm{O}(10)$ & $91.82(14)$ \\
\hline $\mathrm{O}(1)-\mathrm{Ni}(1)-\mathrm{N}(4)$ & $96.17(14)$ & $\mathrm{O}(10)-\mathrm{Ni}(1)-\mathrm{O}(2)$ & $177.73(13)$ \\
\hline $\mathrm{O}(6)-\mathrm{Ni}(1)-\mathrm{N}(4)$ & $172.68(14)$ & $\mathrm{N}(1)-\mathrm{Ni}(1)-\mathrm{N}(4)$ & $84.09(15)$ \\
\hline $\mathrm{O}(2)-\mathrm{Ni}(1)-\mathrm{N}(4)$ & $88.45(15)$ & $\mathrm{Ni}(1)-\mathrm{O}(1)-\mathrm{Ni}(2)$ & $117.96(15)$ \\
\hline
\end{tabular}

present in the complex, monodentate and bidentate bridging modes. The bidentate carboxylates have syn-syn configurations. The binuclear $\mathrm{Ni}(\mathrm{II})$ atoms $(\mathrm{Ni} 1, \mathrm{Ni} 2)$ are bridged by the $\mathrm{O} 1$ from aqua ligand and four oxygen atoms (O6, O7, O10, O11) from two different nba. The Ni-O-Ni linkage is nonlinear, exhibitting an angle of $117.96(15)^{\circ}$ for $\mathrm{Ni}(1)-\mathrm{O}(1)$ $\mathrm{Ni}(2)$.
TABLE-1

EEFINMENT FOR COMPLEX 1

\begin{tabular}{cc} 
Formula weight & 1078.37 \\
Crystal system & Monoclinic \\
$\alpha\left(^{\circ}\right)$ & 90 \\
$\beta\left(^{\circ}\right)$ & $111.310(4)$ \\
$\gamma\left({ }^{\circ}\right)$ & 90 \\
$\mathrm{Z}$ & 4 \\
Absorption coefficient $\left(\mathrm{mm}^{-1}\right)$ & 0.874 \\
$\theta$ Range $\left(^{\circ}\right)$ & $1.73-28.24$ \\
Reflection collected/unique & $52127 / 10855\left[\mathrm{R}_{\text {(int) }}=0.0912\right]$ \\
Goodness-of-fit & 1.059 \\
$\mathrm{R}$ indices (all data) & $\mathrm{R}_{1}=0.1213, \mathrm{wR}_{2}=0.2192$ \\
- & - \\
\hline
\end{tabular}

\section{REFERENCES}

1. Sadiq-ur-Rehman, S.S. Kiani, M.A. Choudhary, K.A. Yasin and M. Safdar, Asian J. Chem., 23, 1497 (2011).

2. H. Aghabozorg, E. Sadr-Khanlou, A. Shokrollahi, M. Ghaedi and M. Shamsipur, J. Iran. Chem. Soc., 6, 55 (2009).

3. A.F. Kolodziej, Prog. Inorg. Chem., 41, 493 (1991).

4. J. Wang, H. Xu, H. Zhou and P. Wei, Chin. J. Struct. Chem., 24, 909 (2005).

5. M. Li, Z. Xu, X. You, Z. Dong and G. Guo, Polyhedron, 12, 921 (1993).

6. A. Altomare, M.C. Burla, M. Camalli, G. Cascarano, C. Giacovazzo, A. Guagliardi, A.G.G. Moliterni, G. Polidori and R. Spagna, J. Appl. Cryst., 32, 115 (1999).

7. G.M. Sheldrick, Acta Cryst., A64, 112 (2008). 